\title{
ANÁLISE ESTATÍSTICA DO DESEMPENHO BALÍSTICO DA BLINDAGEM MULTICAMADA COM COMPÓSITO DE EPÓXI REFORÇADO COM FIBRA DE JUTA*
}

Fernanda Santos da $L u z^{1}$ Sérgio Neves Monteiro²

\section{Resumo}

Atualmente, os sistemas de blindagem multicamada (SBMs) contra munições de alto impacto são constituídos de uma camada frontal cerâmica seguida de tecido de aramida $\left(\operatorname{Kevlar}^{\circledR}\right)$. No presente trabalho foi avaliado o desempenho balístico desse sistema de blindagem multicamada convencional e comparado com o SBM utilizando uma camada compósita de epóxi reforçada com fibra de juta, nas configurações de tecido e manta, substituindo a camada de tecido de aramida de mesma espessura. Os testes balísticos foram realizados de acordo com norma internacional de proteção individual (NIJ 0101.06), utilizando a munição de calibre $7,62 \mathrm{~mm}$ e o desempenho balístico foi avaliado por meio da medida da profundidade média da deformação da plastilina. Para a comparação dessas médias obtidas para cada SBM foi aplicada a análise estatística pelo Teste de Tukey a partir do qual pôde-se afirmar, com um nível de significância de 5\%, que o SBM utilizando a placa compósita de epóxi reforçada com manta de juta, como camada intermediária, teve melhor desempenho balístico que o compósito de epóxi-tecido de juta e que o tecido de aramida.

Palavras-chave: Blindagem; Compósito; Fibra; Juta.

\section{STATISTIC ANALYSIS OF BALLISTIC TEST OF MULTILAYERED ARMOR WITH INTERMEDIATE EPOXY COMPOSITE REINFORCED WITH JUTE FIBER Abstract}

Multilayered Armor Systems (MASs) against high impact ammunitions currently are made with a front ceramic layer followed by aramid fabric (Kevlar ${ }^{\mathrm{TM}}$ ). In the present work it was measured the ballistic performance for this conventional MAS and compared with the MAS with intermediate layer of epoxy composite reinforced with jute fiber. Both jute fabric and sliver shapes, replaced the aramid fabric layer with same thickness. Ballistic tests were carried out according to NIJ 0101.06 standard, using $7.62 \mathrm{~mm}$ caliber ammunition and the ballistic performance was measured through the average depth deformation of clay witness. For comparisons of means for each MAS was used the statistic analysis tested by Tukey's multiple range test from which could be affirmed, with $5 \%$ significance level, that the MAS with epoxy composite reinforced with jute sliver had better ballistic performance than the other intermediate layers.

Keywords: Armor; Composite; Fiber; Jute.

1 Engenheira de Materiais, M.C, Doutoranda em Ciência dos Materiais, Seção de Engenharia Mecânica e de Materiais, Instituto Militar de Engenharia, Rio de Janeiro, RJ, Brasil. fsl.santos@gmail.com

2 Engenheiro Metalurgista, Ph.D, Professor Titular, Seção de Engenharia Mecânica e de Materiais, Instituto Militar de Engenharia, Rio de Janeiro, RJ, Brasil. 


\section{INTRODUÇÃO}

Devido aos crescentes conflitos armados no mundo, têm-se aumentado a pesquisa e o desenvolvimento de novos materiais para proteção balística de forma a otimizar o seu desempenho.

Os sistemas de blindagem multicamada (SBMs) são usualmente empregados para proteção pessoal contra munição de alto impacto, tal como, a de calibre 7,62 mm. Esses sistemas são, geralmente, compostos de: um revestimento frontal de cerâmica, o qual tem a função de erodir e fragmentar o projétil absorvendo grande parte da energia de impacto por meio da fragmentação dinâmica [1]; seguido de um material mais leve, como o tecido de aramida $\left(\operatorname{Kevlar}^{\circledR}\right)$, o qual tem o objetivo de absorver parte da energia cinética proveniente dos fragmentos formados pela camada cerâmica e projétil.

Nesse contexto, diversos estudos têm sido conduzidos para a utilização de diferentes materiais como segunda camada desses SBMs. Primeiramente, as pesquisas foram focadas na aplicação de fibra de vidro [2,3] e compósitos de fibra de carbono [4,5] para a segunda camada. Porém, diversos materiais já têm sua aplicação consolidada em blindagens, tais como, os tecidos de aramida (Kevlar ${ }^{\circledR} \mathrm{e}$ Twaron $\left.^{\circledR}\right)[6,7]$ e as fibras de polietileno de ultra alto peso molecular PEUHPW (Sprectra $^{\circledR}$ e Dyneema $\left.{ }^{\circledR}\right)[8,9]$.

Nos SBMs os pulsos de energia propagam-se nas interfaces entre os diferentes materiais ou na forma de uma onda compressiva, que é refletida como uma onda de tração ou trativa, que depende das impedâncias das camadas, as quais estão diretamente relacionadas com suas densidades [10]. Teoricamente, uma menor energia será transmitida caso o material da segunda camada possua menor impedância de choque que o material da primeira, pois o pulso de compressão atuante será menor. Portanto, a utilização de um material mais leve na segunda camada, tal como, um compósito reforçado com fibras naturais, as quais apresentam menores densidades e baixo custo [11], deve, em princípio, aumentar a absorção da energia de impacto.

No presente trabalho, foi avaliado o desempenho balístico de diferentes SBMs utilizando como camada intermediária ou um tecido de aramida (controle), ou compósito de epóxi reforçado com tecido de juta, ou compósito de epóxi reforçado com manta de juta. Os testes balísticos foram conduzidos conforme a norma NIJ 0101.06 [12] e o desempenho balístico de cada SBM foi comparado pela análise estatística do Teste de Tukey.

O Teste de Tukey também conhecido como teste da diferença honestamente significativa (HSD) avalia a igualdade entre os tratamentos e trata-se de um teste de hipóteses. Então, a partir dos resultados rejeita-se ou não a hipótese de igualdade com base na diferença mínima significativa (d.m.s), dada pela Equação 1:

$$
\text { d.m.s. }=q \cdot \sqrt{\frac{Q M R}{r}}
$$

onde $q$ é amplitude total estudantizada, a qual é função do grau de liberdade (G.L.) do resíduo e do número de tratamentos; QMR é o quadrado médio do resíduo; e r é o número de repetições de cada tratamento.

Além disso, pode-se também verificar a hipótese de igualdade através do P-valor, o qual indica o nível descritivo do teste, sendo rejeitada quando o valor obtido para Pvalor é menor que o nível de significância estabelecido [13]. 


\section{MATERIAIS E MÉTODOS}

Na Figura 1 está ilustrado o tipo de SBM utilizado no presente trabalho, constituído de: camada frontal cerâmica (A), com $10 \mathrm{~mm}$ de espessura; camada intermediária (B) ou de tecido de aramida (i), fornecida pela empresa LFJ Blindagem Com. Serv. S.A., ou compósito de epóxi-tecido de juta (ii) ou compósito de epóxi-manta de juta (iii), também de $10 \mathrm{~mm}$ de espessura; seguida por uma placa de alumínio 5052 H34 (C) de $5 \mathrm{~mm}$ de espessura.

O corpo cerâmico foi fabricado através da sinterização, a $1400^{\circ} \mathrm{C}$, da mistura do pó de $\mathrm{Al}_{2} \mathrm{O}_{3}\left(96 \%\right.$ ), fornecido pela empresa Treibacher Schleifmittel, e do pó de $\mathrm{Nb}_{2} \mathrm{O}_{5}$ $(4 \% p)$ fornecido pela CBMM, prensada uniaxialmente. A rota de sinterização utilizada foi descrita por Trindade et al [14]. Para a produção da matriz epoxídica foram misturados os componentes de bisfenol-A (DGEBA) e endurecedor trietileno tetramina (TETA) com phr 13, fornecidos pela empresa Resinpoxy. Essa matriz foi reforçada com $30 \%$ em volume de fibras de juta, tanto na forma de manta quanto em tecido, e curada sob pressão, em temperatura ambiente, por 24 horas.

A união das três camadas do SBM foi feita por uma fina camada da cola de cura rápida da marca Ultraflex.

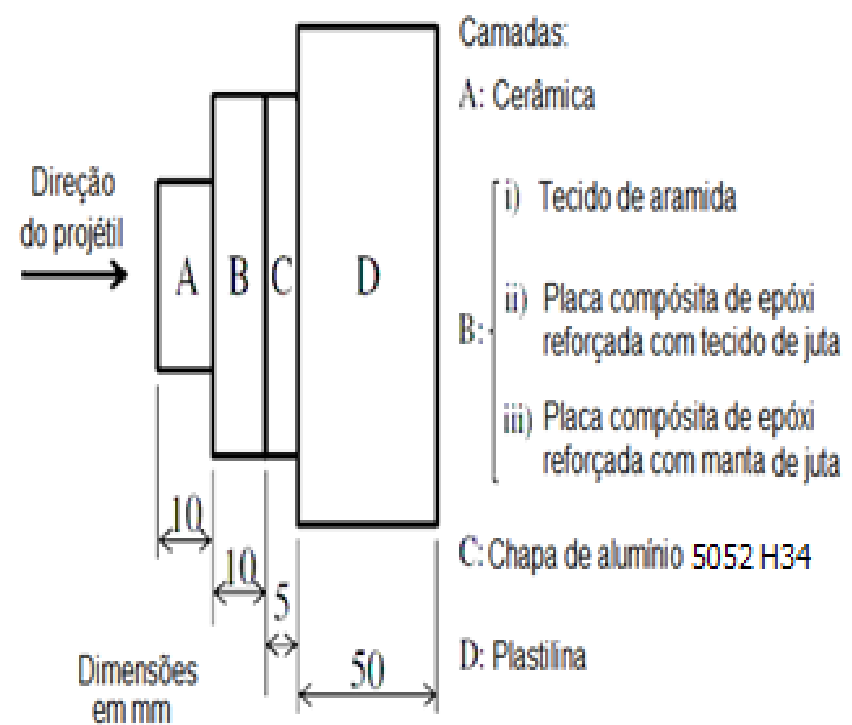

(a)

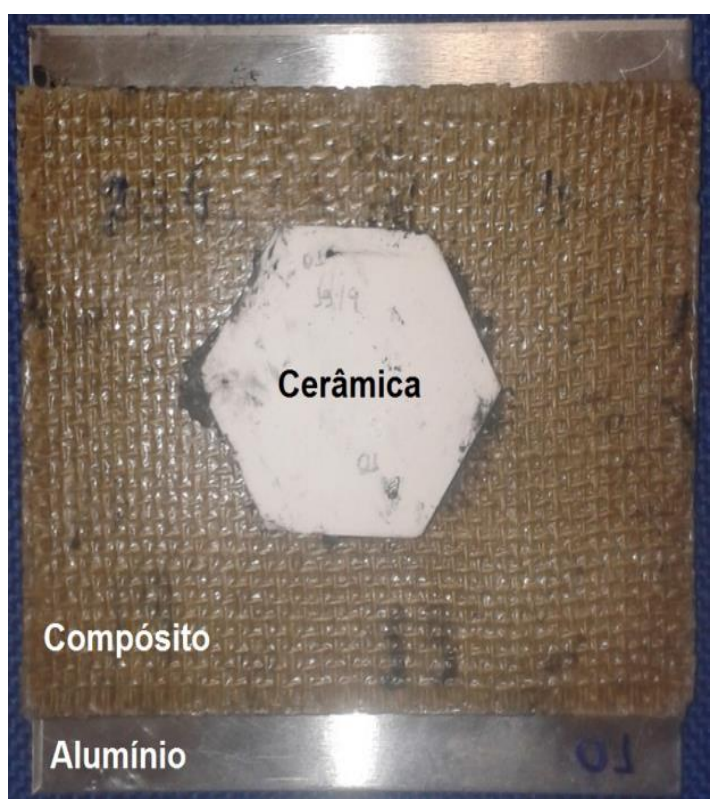

(b)

Figura 1. Sistema de blindagem multicamada (estrutura final do compósito): (a) esquemático; (b) utilizado no presente estudo.

Os ensaios balísticos foram realizados utilizando munição de calibre $7,62 \mathrm{~mm}$ e apoiando o SBM na plastina, material que simula a consistência do corpo humano. $O$ desempenho balístico dos diferentes SBMs foi avaliado conforme a NIJ 0101.06, a qual determina eficiente uma blindagem quando a profundidade do trauma na plastilina é igual ou inferior a $44 \mathrm{~mm}$ [12].

Com o objetivo de comparar os desempenhos balísticos dos SBMs utilizando diferentes camadas intermediárias foi realizada a análise estatística através do Teste de Tukey para comparação das profundidades médias de trauma, partindo dos pressupostos: distribuição normal e homogeneidade de variâncias, os quais foram verificados por meio do teste de normalidade e teste de Hartley. 


\section{RESULTADOS E DISCUSSÃO}

Em todos os testes balísticos realizados na plastilina não houve a perfuração do alvo, consequentemente, o projétil foi parado e a sua energia cinética dissipada no interior do sistema de blindagem multicamada em associação com a indentação (trauma) causada na plastilina. Os três materiais testados como camada intermediária no SBM exibiram uma profundidade de trauma abaixo do limite de $44 \mathrm{~mm}$ estabelecido pela norma NIJ 0101.06 [12].

Os resultados obtidos nos ensaios balísticos estão apresentados na Tabela 1, na qual consta as profundidades médias de trauma na plastilina para cada SBM testado, utilizando diferentes camadas intermediárias. Para verificar se existia alguma diferença significativa entre os desempenhos balísticos dos diferentes SBMs e se os fatores (camada intermediária) exerciam influência na profundidade de trauma (variável dependente) foi realizada a Análise de Variância (ANOVA) desses dados, a qual está mostrada na Tabela 2. Além disso, a diferença entre as médias obtidas foi avaliada pelo Teste de Tukey. O nível de significância selecionado para essas análises foi de $5 \%$ (P.valor $<0,05)$.

Tabela 1. Valores das profundidades médias de trauma na plastilina para os SBMs utilizando diferente tipos de camada intermediária.

\begin{tabular}{|c|c|c|c|c|}
\cline { 3 - 5 } \multicolumn{2}{c|}{} & \multicolumn{3}{c|}{ Camada Intermediária } \\
\cline { 2 - 5 } \multicolumn{2}{c|}{} & Tecido de Juta & Manta de Juta & Aramida \\
\hline \multirow{4}{*}{ Trauma (mm) } & 1 & 18,02 & 14,07 & 17,56 \\
\cline { 2 - 5 } & 2 & 18,78 & 15,07 & 21,07 \\
\cline { 2 - 5 } & 3 & 19,55 & 17,25 & 21,79 \\
\cline { 2 - 5 } & 4 & 20,35 & 17,63 & 23,16 \\
\cline { 2 - 5 } & 5 & 23,15 & 18,02 & 24,98 \\
\cline { 2 - 5 } & 6 & 23,55 & 18,43 & 25,04 \\
\cline { 2 - 5 } & 7 & 24,19 & 20,41 & $\mathbf{2 2 , 6 7}$ \\
\hline Média & & $\mathbf{2 1 , 0 8}$ & $\mathbf{1 7 , 2 7}$ & \\
\hline
\end{tabular}

Tabela 2. Análise de variância das profundidades médias de trauma obtidas para as diferentes camadas intermediárias dos SBMs.

\begin{tabular}{|c|c|c|c|c|c|}
\cline { 2 - 5 } \multicolumn{1}{c|}{} & G.L. & $\begin{array}{c}\text { Soma de } \\
\text { Quadrados }\end{array}$ & $\begin{array}{c}\text { Quadrado } \\
\text { Médio }\end{array}$ & Estat. F & P.valor \\
\hline Fator & 2 & 108,00892 & 54,00446 & \multirow{2}{*}{8,74519} & 0,00222 \\
\hline Resíduos & 18 & 111,15600 & 6,17533 & \\
\hline
\end{tabular}

A partir dos resultados obtidos na análise de variância (Tabela 2) rejeita-se a hipótese de que as médias são iguais com nível de significância de 5\%, pois P-valor calculado foi de 0,00222 , isto é, $\mathrm{P}$-valor $<0,05$. Portanto, as camadas intermediárias têm efeitos diferentes nos sistemas de blindagem multicamada.

Por isso, as diferenças do desempenho balístico dos SBMs com distintas camadas intermediárias foram comparadas para verificar qual SBM teve melhor desempenho ou se estes tiveram resultados similares. Para isso, aplicou-se o Teste de Tukey 
para comparação de médias utilizando um nível de confiança de 95\% (P-valor < 0,05). Os resultados obtidos nessa análise estão apresentados na Tabela 3.

Tabela 3. Comparações múltiplas pelo Teste de Tukey.

\begin{tabular}{|c|c|c|c|c|}
\hline Níveis & Diferença & P-valor & q & d.m.s. \\
\hline Manta-Aramida & $-5,404286$ & 0,00198 & & \\
\cline { 1 - 3 } Tecido-Aramida & $-1,588571$ & 0,47062 & \multirow{2}{*}{3,01} & 2,83 \\
\hline Tecido-Manta & 3,815714 & 0,02609 & & \\
\hline
\end{tabular}

Com base nesses resultados, têm-se que, com nível de significância de 5\%, o SBM com camada intermediária de compósito epóxi e manta de juta apresentou melhor desempenho balístico, pois exibiu menor valor de profundidade média de trauma $(17,27 \mathrm{~mm})$ e esse é significativamente diferente dos demais SBMs, pois o P-valor é menor que $5 \%$, assim como os valores das diferenças são maiores que o valor de d.m.s. Já o SBM com tecido de aramida como camada intermediária apresentou menor desempenho balístico, embora esse não seja significativamente diferente do SBM com a camada compósita de tecido de juta.

Uma possível justificativa para esses resultados pode ser atribuída a menor densidade do compósito epóxi-juta comparado ao tecido de aramida, que em princípio, quanto menor a densidade maior deve ser a redução da energia de impacto, pois uma menor impedância de choque da camada intermediária transmitirá uma menor energia do pulso de compressão [10]. Além disso, a matriz frágil de epóxi participa na dissipação da energia através da nucleação e propagação de trincas. Assim como, outros mecanismos que contribuem para a dissipação de energia, tais como, delaminação, descolamento, rompimento das fibras e captura dos fragmentos gerados pelo impacto do projétil.

\section{CONCLUSÃO}

Os três tipos de materiais testados como camada intermediária no SBM atenderam a norma internacional NIJ 0101.06, ou seja, a profundidade média do trauma foi abaixo do limite de $44 \mathrm{~mm}$ especificado em norma.

Dentre os materiais testados, através do Teste de Tukey, com nível de confiança de 95\%, o SBM com compósito de epóxi e manta de juta apresentou melhor desempenho balístico, com uma profundidade média de indentação de 17,27 mm. Já a aramida exibiu o menor desempenho balístico, com uma profundidade média de indentação de $22,67 \mathrm{~mm}$, a qual não apresenta diferença significativa do resultado obtido para o SBM com compósito de epóxi-tecido de juta.

\section{Agradecimentos}

Os autores deste trabalho agradecem ao CNPq, à FAPERJ e à CAPES por meio do PDS pelo financiamento da pesquisa, através do programa $31007015006 \mathrm{P} 4$, e ao CAEx pela execução dos ensaios balísticos.

\section{REFERÊNCIAS}

1 MEDVEDOVSKI, E. Ballistic Performance of Armour Ceramics: Influence of Design and Structure-Part 1. Ceramics International. 2010; 36: 2103-2115. 
2 CHOU, S.C.; DeLUCA, E.; PRIFTI, J. BETHENEY, W. Ballistic impact damage of S2glass- reinforced plastic structural armor. Composites Science and Technology. 1998; 58: 1453-1461.

3 D'ALMEIDA, J.R.M.; NUNES, L.M.; PACIORNIK, S. Evaluation of the damaged area of glass-fiber-reinforced epoxy-matrix composite materials submitted to ballistic impacts. Composites Science and Technology. 2004; 64: 945-954.

4 VAIDYA, U.K., ULVEN, C.; HOSUR, M.V. Effect of projectile shape during ballistic perforation of VARTM carbon/epoxy composite panels. Composite Structures. 2003; 61(1-2): 143-150.

5 HOSUR, M.V.; VAIDYA, U.K.; ULVEN, C.; JEELANI, S. Performance of stitched/unstitched woven carbon/epoxy composites under high velocity impact loading. Composite Structures. 2004; 64: 455-466.

6 LEE, Y.S.; WETZEL, E.D.; WAGNER, N.J. The Ballistic Impact Characteristic of Kevlar Woven Fabrics Impregnated with a Colloidal Shear Thickening Fluid. Journal of Materials Science. 2003; 38: 2825-2833.

7 JACOBS, M.J.N.; J.L.J.V. DINGENEN. Ballistic protection mechanisms in personal armour. Journal of Materials Science. 2001; 36: 3137-3142.

8 LEE, B. L.; SONG, J. W.; WARD, J. E. Failure of Spectra® Polyethylene FiberReinforced Composites Under Ballistic Impact Loading. Journal of Composite Materials. 1994; 28 (13): 1202-1226.

9 MORYE, S.S.; HINE, P.J.; DUCKETT, R.A.; CARR, D.J.; WARD, I.M. Modelling of the energy absorption by polymer composites upon ballistic impact. Compos Sci Technol. 2000; 60: 2631-2642.

10 MEYERS, M.A. Dynamic Behavior of Materials. John Wiley \& Sons, New York, 1994.

11 MONTEIRO, S.N. et al. Natural-Fiber Polymer-Matrix Composites: cheaper, tougher, and environmentally friendly - An overview. JOM - A Publication of The Minerals, Metals \& Materials Society; Vol. 61, nำ1, pp.17-22, 2009.

12 NIJ Standard 0101.04. Ballistic Resistance of Personal Body Armor. U.S. Department of Justice/Office of Justice Programs - National Institute of Justice. 2000.

13 RODRIGUES, M.I.; IEMMA, A.F. Planejamento de Experimentos e Otimização de Processos. 3a Edição. Campinas, SP: Casa do Espírito Amigo Fraternidade Fé e Amor; 2014.

14 TRINDADE, W.; GOMES. A. V.; LOURO, L.H.L. Elaboração de uma Nova Rota de Eliminação de Ligante da Cerâmica De Alumina. Revista Militar de Ciência \& Tecnologia. 2013; 4: 71-79. 